\title{
Development of a determination method of the prompt $\gamma$-ray emission probability for the measurement of neutron capture cross sections
}

\author{
I. Miyazaki ${ }^{1, a}$, H. Hayashi ${ }^{1}$, A. Tojo ${ }^{1}$, A. Taniguchi $^{3}$, and M. Shibata ${ }^{2}$ \\ 1 Graduate School of Engineering, Nagoya University, Nagoya 464-8603, Japan \\ 2 Radioisotope Research Center, Nagoya University, Nagoya 464-8602, Japan \\ 3 Research Reactor Institute, Kyoto University, Kumatori 590-0494, Japan
}

\begin{abstract}
We propose a promising method of the determination of the emission probabilities by normalizing the sum of the relative intensities of the $\gamma$-rays populating the ground state. The prompt $\gamma$-rays from thermal-neutron capture in ${ }^{23} \mathrm{Na},{ }^{27} \mathrm{Al},{ }^{51} \mathrm{~V},{ }^{55} \mathrm{Mn},{ }^{59} \mathrm{Co},{ }^{141} \mathrm{Pr},{ }^{186} \mathrm{~W}$ and ${ }^{197} \mathrm{Au}$ were measured and the emission probabilities by this method were deduced. The experiment was performed at the supermirror neutron guide tube at the Kyoto University Reactor. For the nucleus ${ }^{24} \mathrm{Na}$, the emission probabilities were deduced and they were in agreement with the values, which were deduced by using the $\gamma$-rays following the $\beta$-decay, within the uncertainties of approximately $5 \%$.
\end{abstract}

\section{Introduction}

Nuclear power generation has generated radioactive nuclear wastes consisting mainly of long-lived fission products (LLFPs). Nuclear transmutation is a proposed method for reducing LLFPs by transmuting them to stable or short-lived ones. One of the transmuting methods uses a neutron capture reaction, which requires accurate and reliable experimental cross-section data for precise monitoring of the transmutation chain. These data are also needed for a wide energy range. Prompt $\gamma$-rays from neutron capture are useful for measuring the cross sections because these rays are always emitted from the produced nuclides regardless of nuclides' stability.

The precision of the $\gamma$-ray emission probabilities is important for the measurement of neutron-capture cross sections. Assuming that all $\gamma$ rays emitted in the reaction are observed and a complete level scheme is constructed, the following relations hold:

$$
\sum E_{\gamma} I_{\gamma} / S_{n}=\sum I_{\gamma, c}=\sum I_{\gamma, g}=100 \%
$$

where $E_{\gamma}$ indicates the $\gamma$-ray energy, $I_{\gamma}$ indicates the emission probability in consideration of internal conversion and $S_{n}$ indicates the neutron separation energy, which is equal to the energy gap between the capture state to the ground state. The subscript $c$ and $g$ indicate depopulating the capture state and populating the ground state, respectively. The emission probability is normalized using the relation in equation (1). When not all emitted $\gamma$ rays are measured, the normalized emission probability is the upper limit.

Prompt $\gamma$-ray emission probabilities can be deduced the other method. The $\gamma$-ray emission probability can be normalized to the intensity per one capture by using the $\gamma$-ray following the $\beta$-decay as shown in figure 1 and is given by the

\footnotetext{
${ }^{a}$ Presenting author, e-mail: h052305d@mbox.nagoya-u.ac . jp
}

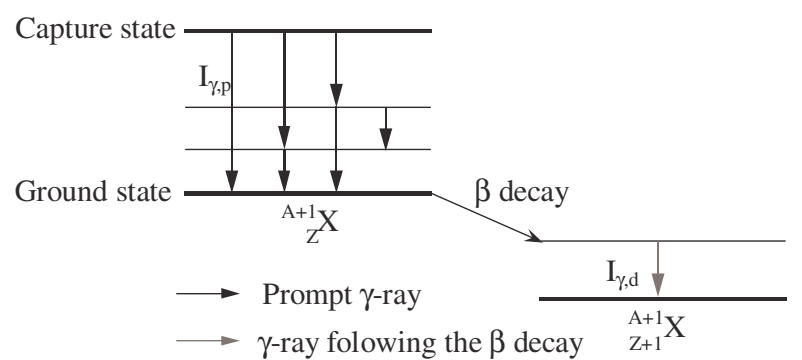

Fig. 1. Schematic views of a level scheme and a decay scheme. $\mathrm{I}_{\gamma, \mathrm{p}}$ and $\mathrm{I}_{\gamma, \mathrm{d}}$ indicate the prompt $\gamma$-ray emission probability and the emission probability of the $\gamma$-ray following the $\beta$ decay, respectively.

following equation:

$$
I_{p}=\frac{C_{p} \cdot \varepsilon_{d}}{C_{d} \cdot \varepsilon_{p}} \cdot T(t) \cdot I_{d}
$$

where $C$ indicates is the $\gamma$-ray peak count, $I$ is the emission probability, $\varepsilon$ is the relative efficiency of a $\gamma$-ray of interest. $T(t)$ indicates the function of time. The subscripts $p$ and $d$ indicate a prompt $\gamma$-ray and a $\gamma$-ray following the $\beta$ decay, respectively.

We aimed to develop a measurement method for emission probability by using the prompt $\gamma$-rays populating the ground state. In this work, the prompt $\gamma$-rays from thermal-neutron capture in ${ }^{23} \mathrm{Na},{ }^{27} \mathrm{Al},{ }^{51} \mathrm{~V},{ }^{55} \mathrm{Mn},{ }^{59} \mathrm{Co},{ }^{141} \mathrm{Pr},{ }^{186} \mathrm{~W}$ and ${ }^{197} \mathrm{Au}$ were measured. These nuclides become radioactive ones and emit $\gamma$-rays following the $\beta$ decay after neutron capture. The prompt $\gamma$-ray emission probabilities deduced by using the prompt $\gamma$-rays and the $\gamma$-rays following the $\beta$ decay were compared with each other.

\section{Experimental}

\subsection{Neutron source}

Neutron irradiations were carried out by the supermirror neutron guide tube at the Kyoto University Reactor [1]. 


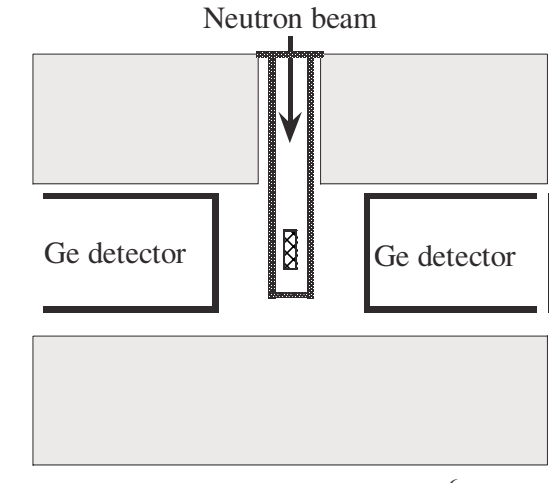

Sample $\square$ Lead $\square{ }^{6} \mathrm{LiF}$

Fig. 2. A schematic view of the present experimental arrangement.

The dimension of the beam is about $14 \mathrm{~mm}$ in width and $61 \mathrm{~mm}$ in height. The typical thermal neutron flux at the irradiation position was $1.6 \times 10^{7} \mathrm{n} / \mathrm{sm}^{2} / \mathrm{s}$ at $5 \mathrm{MW}$ operations.

\subsection{Detector and sample preparation}

The $\gamma$-ray measurements were performed using $22 \%$ and $36 \%$ HPGe detectors. Both distances from the sample to the detectors were $10.0 \mathrm{~cm}$. The detection efficiencies at the same position were determined using ${ }^{152} \mathrm{Eu}$ [2] and the prompt $\gamma$-rays from the ${ }^{14} \mathrm{~N}(\mathrm{n}, \gamma){ }^{15} \mathrm{~N}$ [3]. The uncertainties in the efficiency curves were evaluated to be within $0.7 \%$ between 0.244 and $5 \mathrm{MeV}$ and $1.0 \%$ above $5 \mathrm{MeV}$. The experimental arrangement is shown in figure 2. The target material for ${ }^{186} \mathrm{~W}(\mathrm{n}, \gamma){ }^{187} \mathrm{~W}$ was a separated isotope and the others were materials found abundantly in nature. Powder samples were wrapped in waxed paper, and were $0.1-1.5 \mathrm{~mm}$ thick. Metal samples were 0.2 and $0.05 \mathrm{~mm}$ thick and rectangular-shaped $(10 \mathrm{~mm} \times 10 \mathrm{~mm})$. Table 1 lists the samples' isotopic abundance [4], chemical form, and weight. The samples were located on a holder made of carbon plates, and they were set at $30^{\circ}$ to each detector in the target box so as to receive the most intense part of the neutron beam. The target box which was made of enriched lithium fluoride (having a $10 \mathrm{~mm}$ wall thickness) was used as a thermal neutron absorber in order to reduce the background $\gamma$-rays from neutron capture in the surrounding materials.

\subsection{Measurements}

A schematic view of measuring processes is shown in figure 3 . During irradiation, prompt $\gamma$-rays were measured. After the irradiation had ceased, $\gamma$-rays following the $\beta$-decay from the induced activities were measured. A beam shutter made of enriched lithium fluoride was used for stopping the irradiation. Typical $\gamma$-ray spectra of the ${ }^{27} \mathrm{Al}(\mathrm{n}, \gamma){ }^{28} \mathrm{Al}$ are shown in figure 4 , demonstrating that the seven prompt $\gamma$-rays in spectrum (a) cannot be observed in spectrum (b). The $1778 \mathrm{keV} \gamma$-ray, which is the $\gamma$-ray following the $\beta$-decay, are obserbed in spectrum (a), because the half-life of ${ }^{28} \mathrm{Al}$ is much shorter than the measuring time. The shutter made of lithium fluoride
Table 1. Abundance, chemical form thickness, and weight of the samples.

\begin{tabular}{lccc}
\hline $\begin{array}{l}\text { Target } \\
\text { nuclide }\end{array}$ & $\begin{array}{c}\text { Abundance } \\
{[\%]}\end{array}$ & Chemical form & $\begin{array}{c}\text { Weight } \\
{[\mathrm{mg}]}\end{array}$ \\
\hline${ }^{23} \mathrm{Na}$ & 100 & $\mathrm{NaF}$ (Powder) & 176.1 \\
${ }^{27} \mathrm{Al}$ & 100 & $\mathrm{Al}$ (Metal) & 102.8 \\
${ }^{51} \mathrm{~V}$ & 99.750 & $\mathrm{~V}_{2} \mathrm{O}_{5}$ (Powder) & 90.0 \\
${ }^{55} \mathrm{Mn}$ & 100 & $\mathrm{Mn}$ (Powder) & 49.7 \\
${ }^{59} \mathrm{Co}$ & 100 & $\mathrm{Co}$ (Metal) & 104.3 \\
${ }^{141} \mathrm{Pr}$ & 100 & $\mathrm{Pr}_{6} \mathrm{O}_{11}$ (Powder) & 71.0 \\
${ }^{186} \mathrm{~W}$ & $97.5^{\mathrm{a}}$ & $\mathrm{WO}_{3}$ (Powder) & 68.8 \\
${ }^{197} \mathrm{Au}$ & 100 & $\mathrm{Au}$ (Metal) & 18.4 \\
\hline
\end{tabular}

${ }^{a}$ Separated isotope sample.

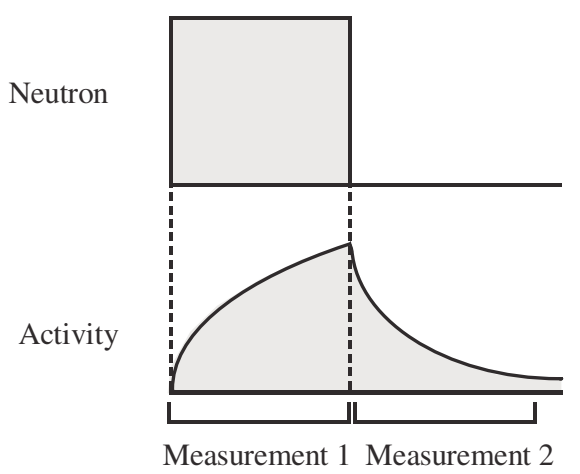

Fig. 3. A schematic view of the measuring process. Prompt $\gamma$-rays are measured in "Measurement 1". Gamma-rays following the $\beta$-decay are measured in "Measurement 2".

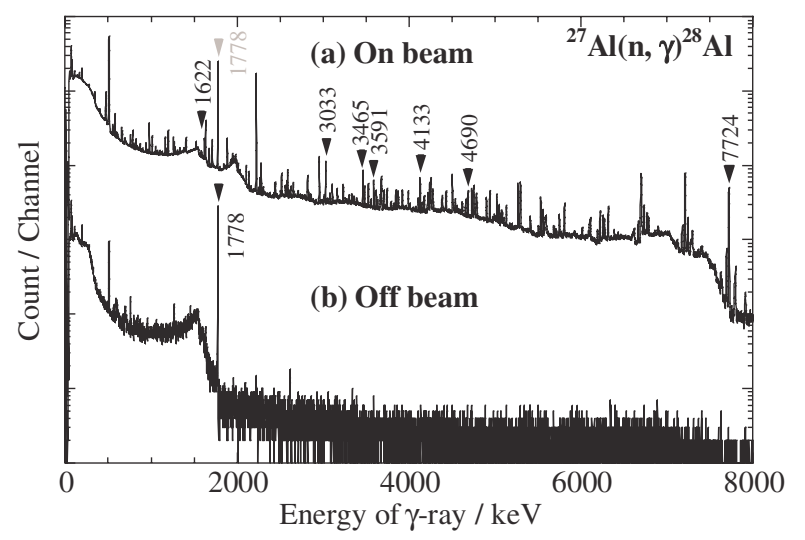

Fig. 4. Spectra for capture of thermal neutrons in ${ }^{27} \mathrm{Al}$ with (a) on beam and (b) off-beam. The seven prompt $\gamma$-rays in the spectrum of (a) are not observed in the spectrum of (b).

worked for stopping the neutron irradiation. The neutron penetration was deduced at $2.7 \times 10^{-6}$ and was negligibly small.

\section{Results}

The prompt $\gamma$-ray emission probabilities of ${ }^{23} \mathrm{Na}(\mathrm{n}, \gamma)$ and ${ }^{27} \mathrm{Al}(\mathrm{n}, \gamma)$ were deduced by using the sum of relative intensities of the prompt $\gamma$-rays depopulating to the ground state from 

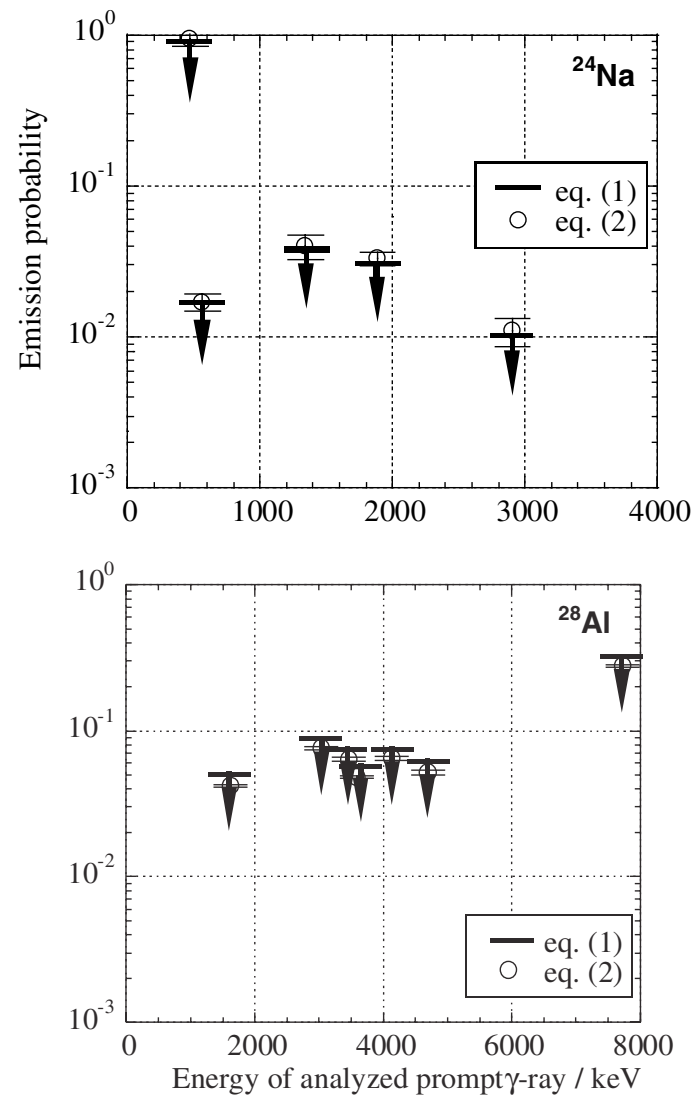

Fig. 5. Comparisons between the results by using the prompt $\gamma$-rays, as shown equation (1), and those by using the $\gamma$-ray following the $\beta$ decay, as shown equation (2). A descending arrow is an upper limit value.

equation (1). Eight $\gamma$-rays were used for the analysis for ${ }^{23} \mathrm{Na}(\mathrm{n}, \gamma)$. On the other hand, $48 \gamma$-rays, including the $\gamma$ rays depopulating $30.63 \mathrm{keV}$ level, were used for ${ }^{27} \mathrm{Al}(\mathrm{n}, \gamma)$. The information of the level scheme refers to the Evaluated Nuclear Structure Data File [5]. The emission probabilities were also deduced by using the $\gamma$-ray following the $\beta$-decay from equation (2). The intensive ones are shown in figure 5 . For ${ }^{23} \mathrm{Na}(\mathrm{n}, \gamma)$, the results by using the prompt $\gamma$-rays are in good agreement with those by using the $\gamma$-ray following $\beta$-decay within the uncertainties of approximately $5 \%$. It is considered that there are few missing $\gamma$-rays in the present measurements. For ${ }^{27} \mathrm{Al}(\mathrm{n}, \gamma)$, the results by using the prompt $\gamma$-rays are larger by a factor of 1.1 than those by using the $\gamma$ ray following $\beta$ decay. The sum of the emission probabilities of the missing $\gamma$-rays in the present measurements can be estimated to be 0.1 .

\section{Summary}

We have concluded that the method of the emission probabilities by normalizing the sum of the relative intensities of the $\gamma$ rays populating the ground state are useful. The present results of ${ }^{23} \mathrm{Na}(\mathrm{n}, \gamma)$ and ${ }^{27} \mathrm{Al}(\mathrm{n}, \gamma)$ are consistent with analysis of the $\gamma$-ray following $\beta$-decay. For other nuclides which have more complex level scheme, we need to check whether the prompt $\gamma$-rays populating the ground state can determine the emission probabilities. For the other nuclei, detailed analysis is now in progress. Additionally, we need a method to estimate absolute values from upper limit values.

We gratefully acknowledge the operators at the Kyoto University Reactor for the neutron irradiation. We are grateful to Dr K. Kawade for his valuable discussions.

\section{References}

1. T. Akiyoshi, T. Ebisawa, T. Kawai, F. Yoshida, M. Ono, S. Tasaki, S. Mitani, T. Kobayashi, S. Okamoto, J. Nucl. Sci. Technol. 29, 939 (1992).

2. X-Ray and Gamma-Ray Standards for Detector Calibration, International Atomic Energy Agency, Vienna, 1991.

3. H. Takayama et al., in Proceedings of the 2004 Symposium on Nuclear Data INDC(JPN)-195/U, edited by Y. Tahara, T. Fukahori (JAERI, Tokai, Japan, 2005), pp. 222-227.

4. R.B. Firestone, V.S. Sirley (John Wiley \& Sons, New York, 1996).

5. ENSDF, Evaluated Nuclear Structure Data File, edited and maintained by the National Nuclear Data Center, Brookhaven National Laboratory, on behalf of the International Network for Nuclear Structure Data Evaluation (August 28, 2006). 